\title{
Correction to: Arctic Hydrology, Permafrost and Ecosystems
}

Daqing Yang and Douglas L. Kane

\section{Correction to: \\ D. Yang and D. L. Kane (eds.), Arctic Hydrology, Permafrost and Ecosystems, https://doi.org/10.1007/978-3-030-50930-9}

In an earlier version of this book, the affiliation of the volume editor "Daqing Yang" was incorrect. This has been corrected. Correct Affiliations is Daqing Yang, Watershed Hydrology and Ecology, Research Division, Water Science and Technology Directorate, Environment and Climate Change Canada, Victoria, BC, Canada. 